\title{
A Disintegrin and Metalloproteases (ADAMs) in Cardiovascular, Metabolic and Inflammatory Diseases: Aspects for Theranostic Approaches
}

\author{
Emiel P. C. van der Vorst ${ }^{1,2}$ Christian Weber ${ }^{1,2,3}$ Marjo M. P. C. Donners ${ }^{1}$ \\ ${ }^{1}$ Cardiovascular Research Institute Maastricht, Maastricht University, \\ Maastricht, The Netherlands \\ 2 Institute for Cardiovascular Prevention, Ludwig-Maximilians- \\ University Munich, Munich, Germany \\ ${ }^{3}$ DZHK (German Centre for Cardiovascular Research), Partner Site

\begin{abstract}
Address for correspondence Marjo M. P. C. Donners, PhD, Department of Pathology, Cardiovascular Research Institute Maastricht, Maastricht University, Universiteitssingel 50, 6229 ER Maastricht, The Netherlands (e-mail: Marjo.donners@maastrichtuniversity.nl).
\end{abstract} Munich Heart Alliance, Munich, Germany

Thromb Haemost 2018;118:1167-1175.

\begin{abstract}
Keywords

- a disintegrin and metalloprotease

- pathologies

- cardiovascular disease

- atherosclerosis

- therapy

A disintegrin and metalloproteases (ADAMs) are membrane-bound enzymes responsible for the shedding or cleavage of various cell surface molecules, such as adhesion molecules, cytokines/chemokines and growth factors. This shedding can result in the release of soluble proteins that can exert agonistic or antagonistic functions. Additionally, ADAM-mediated cleavage can render these membrane proteins inactive. This review will describe the role and association of ADAMs in various pathologies with a main focus on ADAM10 and ADAM17 in atherosclerosis, including a brief overview of atherosclerosis-related ADAM substrates. Furthermore, ADAMs involvement in other metabolic and inflammatory diseases like diabetes, sepsis, Alzheimer's disease and rheumatoid arthritis will be highlighted. Subsequently, we will briefly discuss an interesting emerging field of research, i.e. the potential implications of ADAM expression in extracellular vesicles. Finally, several ADAM-based therapeutic and diagnostic (theranostic) opportunities will be discussed, while focusing on key questions about the required specificity and selectivity.
\end{abstract}

\section{The ADAMs Family: Function and Regulation}

The mammalian genome consists of 40 a disintegrin and metalloprotease (ADAM) family members, although only 12 ADAM genes encode for proteolytically active proteases able to cleave/shed surface molecules, i.e. ADAM8, 9, 10, 12, 15, $17,19,20,21,28,30$ and $33 .{ }^{1}$ ADAM cleavage or shedding can result in the release of soluble factors, such as tumour necrosis factor (TNF), or of intracellular domains like Notch after regulated intra-membrane proteolysis. ${ }^{2}$ Besides the

Note: The review process for this paper was fully handled by Gregory Y. H. Lip, Editor-in-Chief.

metalloproteinase domain, ADAMs also contain a disintegrin domain, capable of binding to various integrins, although this function is still poorly understood. ${ }^{3}$ Especially ADAM10 and ADAM17 are the best studied family members and have been shown to be involved in the shedding of inflammatory mediators like cytokines/chemokines and adhesion molecules. The list of substrates that can be shed by these proteases is substantial and still increasing (reviewed in Dreymueller et al, ${ }^{4}$ van der Vorst et al, ${ }^{5}$ Zhang et al, ${ }^{6}$ and Zunke and Rose-John ${ }^{7}$ ). For ADAM17, one of the best known substrates is TNF, hence the alternative nomenclature for this protease is TNF converting enzyme. ${ }^{8}$

While ADAM expression and activity is regulated at various levels, e.g. at the transcriptional and post-transcriptional level

(c) 2018 Georg Thieme Verlag KG Stuttgart · New York
DOI https://doi.org/ $10.1055 / \mathrm{s}-0038-1660479$. ISSN 0340-6245. 
(reviewed in Dreymueller and Ludwig ${ }^{9}$ and Grötzinger et $\mathrm{al}^{10}$ ), evidence is emerging that substrate-specificity is regulated by membrane localization. ${ }^{11}$ ADAM proteases are produced as latent enzymes, which are activated by removal of the prodomain by furin-like convertases. ${ }^{1}$ Post-transcriptionally, tissue inhibitors of metalloproteases (TIMPs) regulate ADAM activities as TIMP1 blocks ADAM10, whereas TIMP3 inhibits both ADAM10 and ADAM17 activity. ${ }^{12}$ No consensus sequence exists that determines whether these ADAMs cleave a specific substrate. Recently, it has been shown that the surface exposure of phosphatidylserine (PS) is necessary for ADAM17 to exert its sheddase activity. ${ }^{13}$ ADAM17 has been shown to bind to PS and this interaction speculatively directs the protease to its substrate. $^{10,13}$ Increasing evidence suggests that (co-)localization of the enzyme and substrate regulate cleavage activities. Lipid rafts, for example, are cholesterol-rich areas in the membrane where many receptors and signalling molecules cluster together. ${ }^{14}$ Previously, it has been shown that ADAM17 is preferentially located in these rafts. Lipid raft disruption resulted in increased shedding of TNF as disruption brought the ADAM17 protease in close vicinity of its substrate TNF, further confirming a crucial regulatory role of this membrane localization in ADAM activity. ${ }^{15}$ Besides ADAM17, ADAM10 has also been shown to be regulated by lipid raft modulation as cholesterol depletion affected both ADAM10- and ADAM17mediated shedding of Fas ligand. ${ }^{16}$ Besides lipid rafts, also other membrane domains gained interest for ADAMs regulation, i.e. the tetraspanin-enriched micro-domains. For example, ADAM10-mediated amyloid precursor protein (APP) cleavage was shown to be regulated by tetraspanin $12,{ }^{17}$ whereas ADAM10-dependent cleavage of epidermal growth factor is regulated by tetraspanins CD9, CD81 and CD82. ${ }^{18}$ The Tspan C8 family was shown to regulate ADAM10 maturation and trafficking to the cell surface in endothelial cells. ${ }^{19}$ Interestingly, data from Noy et al suggested that different members of this Tspan family regulate substrate selectivity of ADAM10, as further discussed below. ${ }^{20}$ While tetraspanins seem to mainly regulate ADAM10, maturation and trafficking of ADAM17 is specifically regulated by the catalytically inactive family of rhomboid proteases. ${ }^{21-23}$ Research focusing on the regulation of ADAM activity is still a very dynamic and evolving field.

\section{ADAMs in Cardiovascular Disease}

Focusing on cardiovascular disease (CVD) and its underlying pathogenesis of atherosclerosis development, various crucial mediators have already been identified as substrates for ADAM10 and/or ADAM17 (extensively reviewed by van der Vorst et al and Dreymueller et al ${ }^{4,5}$ ). For example, we and others identified the junctional molecules vascular endothelial (VE)cadherin and junctional adhesion molecule $\mathrm{A}$, which play a crucial role in vascular permeability and leukocyte transmigration as ADAM10/17 substrates. ${ }^{24-26}$ Additionally, ADAM10/17 have been shown to shed the platelet receptors glycoprotein I (GPI) and GPVI which are important in thrombus formation, ${ }^{27}$ although the precise impact of ADAM10/17 shedding on thrombosis itself remains controversial. Furthermore, both proteases have been shown to be involved in the cleavage of
$\mathrm{CX}_{3} \mathrm{CL} 1$ and $\mathrm{CXCL} 16$, which are chemotactic proteins that are synthesized as transmembrane molecules with adhesive properties and upon cleavage produce a soluble chemoattractant. Cleavage of these proteins by ADAM10/17 can thus result in a reduction of leukocyte adhesion and even detachment of bound cells, although the attraction of new cells increases by the release of the soluble chemoattractant. ${ }^{28,29}$ Intercellular adhesion molecule 1 (ICAM-1) and vascular cell adhesion molecule 1 (VCAM-1) are two other essential leukocyte adhesion molecules that are critically involved in atherosclerotic lesion initiation/development and that can be shed by ADAM17. ${ }^{30,31}$ Furthermore, ADAM17-mediated shedding of syndecans has been reported which may affect leukocyte recruitment by altering chemokine/cytokine signalling. ${ }^{32,33}$ Importantly, many soluble forms of ADAM substrates, like sICAM, sVCAM, sRAGE (receptor for advanced glycation end-products), SCD40L, sIL6R and TNF, have been shown to correlate with (clinical events of) CVD and were identified as potential biomarkers. ${ }^{34-36}$ Recently, Rizza et al showed a clear correlation between ADAM17 substrates and recurring cardiovascular events in human subjects with atherosclerosis. ${ }^{37}$ Considering their role in inflammation and leukocyte recruitment, ADAMs most likely also play a crucial role in atherosclerosis.

Indeed, several ADAMs have already been associated with atherosclerosis development (-Table 1). For example, the rs653765 polymorphism in the ADAM10 promoter, leading to a significantly enhanced ADAM10 expression, was found to be associated with atherosclerotic cerebral infarction in a Chinese population. ${ }^{38}$ Moreover, we showed ADAM10 expression to be relatively low in a healthy human vessel wall and early human atherosclerotic lesion, but this significantly increased during plaque progression. ${ }^{24}$ As ADAM10 fullbody knockout mice are embryonically lethal, the effect of total ADAM10 deficiency on atherosclerosis cannot be evaluated. However, using myeloid-specific ADAM10-deficient mice we could observe that a myeloid-specific deficiency of ADAM10 did not alter lesion size, but shifted the balance from inflammation towards fibrosis in these atherosclerotic lesions. ${ }^{39}$ Additionally, also ADAM17 has been associated with atherosclerosis development. In mouse quantitative trait locus mapping, increased ADAM17 expression has been shown to be associated with atherosclerosis resistance, ${ }^{40}$ while in rats enhanced ADAM17 expression has been associated with cardiac remodelling after acute myocardial infarction. ${ }^{41}$ Cardiomyocyte ADAM17 has also been shown to be of crucial importance in post-infarction recovery by regulating VE growth factor receptor 2 transcription and angiogenesis, thereby limiting left ventricular dilation and dysfunction. ${ }^{42}$ A recent review by Chute et al provides a more detailed overview of the role of ADAMs in heart physiology and pathology. ${ }^{43}$ Furthermore, Canault et al showed that ADAM17 expression is associated with lesions in atherosclerosis-prone sites in mice and may contribute to elevated levels of soluble TNF receptor in the plasma. ${ }^{44}$ Collectively, these results suggest an important role for ADAM17 in atherosclerosis and CVD, although observed associations are still contradicting. Using lentiviral knockdown of ADAM17 in abdominal aortic plaques of rabbits, Zhao et al showed lower plaque burden with 
Table 1 ADAMs in atherosclerosis/CVD in humans

\begin{tabular}{|c|c|c|}
\hline ADAM proteases & Main conclusion & Reference \\
\hline \multirow[t]{2}{*}{ ADAM8 } & $\begin{array}{l}\text { Single nucleotide polymorphism } 2662 \mathrm{~T} / \mathrm{G} \text { associated with atherosclerosis development } \\
\text { and fatal myocardial infarction }\end{array}$ & 55 \\
\hline & $\begin{array}{l}\text { Polymorphism (rs2275725) is associated with atherosclerosis development and the } \\
\text { occurrence of myocardial infarctions }\end{array}$ & 56 \\
\hline ADAM9 & $\begin{array}{l}\text { Up-regulated in macrophages in advanced human atherosclerotic plaques in samples from } \\
\text { carotid, aortic and femoral territories compared with samples from internal thoracic artery } \\
\text { free of atherosclerotic plaques }\end{array}$ & 50 \\
\hline ADAM10 & $\begin{array}{l}\text { Increased expression during atherosclerotic plaque progression; highly expressed in plaque } \\
\text { micro-vessels and macrophages/foam cells }\end{array}$ & 24 \\
\hline ADAM15 & $\begin{array}{l}\text { Up-regulated in macrophages in advanced human atherosclerotic plaques in samples from } \\
\text { carotid, aortic and femoral territories compared with samples from internal thoracic artery } \\
\text { free of atherosclerotic plaques }\end{array}$ & 50 \\
\hline \multirow[t]{3}{*}{ ADAM17 } & $\begin{array}{l}\text { Correlation between ADAM17 substrates (e.g. sICAM-1 and sTNFR1) and recurring } \\
\text { cardiovascular events in human patients with atherosclerosis }\end{array}$ & 37 \\
\hline & $\begin{array}{l}\text { Up-regulated in macrophages in advanced human atherosclerotic plaques in samples from } \\
\text { carotid, aortic and femoral territories compared with samples from internal thoracic artery } \\
\text { free of atherosclerotic plaques }\end{array}$ & 50 \\
\hline & Detected and active in micro-particles released from atherosclerotic lesions & 84 \\
\hline \multirow[t]{3}{*}{ ADAM33 } & Expressed in human atherosclerotic lesions in smooth muscle cells and leukocytes & 54 \\
\hline & Polymorphism (rs574174) is associated with atherosclerosis development & 54 \\
\hline & $\begin{array}{l}\text { Individuals homozygous for the rs } 2280090 \text { polymorphism have an increased risk of } \\
\text { all-cause and cardiovascular mortality compared with wild types }\end{array}$ & 97 \\
\hline
\end{tabular}

Abbreviations: ADAM, a disintegrin and metalloprotease; CVD, cardiovascular disease; sICAM-1, soluble intercellular adhesion molecule-1; sTNFR1, soluble tumor necrosis factor receptor 1.

reduced expression of inflammatory genes and increased expression of transforming growth factor- $\beta$ (TGF $\beta$ ), indicative of enhanced plaque stability. ${ }^{45}$ Recent studies by Nicolaou et al using ADAM17 hypomorphic mice, expressing very low levels of ADAM $17,{ }^{46}$ could clearly show a atheroprotective role of ADAM17 in atherosclerosis development, which they attributed to the cleaving of membrane-bound TNF and TNF-receptor2 preventing endogenous TNF signalling in vascular cells. ${ }^{47}$ Interestingly, they recently also revealed a function of ADAM17 in arterial elastin network maintenance, although the exact underlying mechanism remains to be elucidated. ${ }^{48}$ We could confirm such atheroprotective effects of ADAM17 in a myeloid-specific ADAM17-deficiency (ADAM17-LysM cre) mouse model. ${ }^{49}$ Remarkably, however, endothelial ADAM17 has contrasting atheroprogressive effects as was shown in ADAM17-Bmx Cre mice. This is more in line with the findings by Zhao et al, as their lentiviral approach most likely targeted especially the endothelium. The exact mechanisms behind the opposing effects on atherosclerosis development remain to be investigated.

Besides ADAM17, ADAM9 and ADAM15 are also up-regulated in macrophages in advanced human atherosclerotic plaques. $^{50}$ For ADAM15 even a causal relation has been described, showing reduced atherosclerosis development in rabbits over-expressing ADAM15. ${ }^{51}$ In sharp contrast, more recently Sun et al showed that ADAM15 deficiency in mice resulted in decreased endothelial permeability, monocyte and neutrophil transmigration and consequently decreased atherosclerotic lesion development. ${ }^{52,53}$ This clearly indicates that ADAM15 plays a crucial role in atherosclerosis development, although the precise mechanisms are still not completely understood. Furthermore, ADAM33 has been shown to be expressed in human atherosclerotic lesions, and polymorphisms of both ADAM33 and ADAM8 genes are associated with atherosclerosis development. ${ }^{54,55}$ In addition, an ADAM8 polymorphism (rs2275725) has been associated with atherosclerosis development and occurrence of myocardial infarctions. ${ }^{56}$ Recently, we could show that ADAM8 expression was up-regulated in vulnerable human plaques, compared with stable lesions and that this expression was predominantly in the active shoulder region of the lesion. ${ }^{57}$ Remarkably, however, neither a hematopoietic nor whole-body ADAM8 deficiency in mice affected atherosclerotic lesion size. ${ }^{57}$

\section{ADAMs in Other Metabolic and Inflammatory Diseases}

Besides a role in CVD and atherosclerosis, ADAMs are also associated with various other metabolic and inflammatory pathologies, like diabetes, sepsis, rheumatoid arthritis and Alzheimer's disease. Especially the role of ADAM17 in diabetes is already thoroughly investigated and nicely reviewed by Menghini et al. ${ }^{58}$ In short, ADAM17 on human white adipocytes has been shown to result in the expression of inflammatory molecules like monocyte chemotactic protein 1 and interleukin 6 (IL-6) and the secretion of soluble IL-6 receptor, ${ }^{59}$ which mediates IL-6 signalling via activation of 
gp130 receptors on cells not expressing the IL-6R themselves (a process called trans-signalling). Interestingly, inhibition of IL-6 trans-signalling reduced atherosclerosis development in mice. ${ }^{60}$ Additionally, ADAM17 on skeletal muscles induces the release of TNF and therefore causes insulin resistance via inhibition of the glucose transport. Finally, ADAM17 present on hepatocytes increases oxidative stress and promotes hepatic steatosis upon hyperinsulinemia. Besides ADAM17, also serum ADAM10 levels, representing extracellular vesicle-associated ADAM10, and its substrate RAGE have been associated with type 1 diabetes in humans. ${ }^{61}$

Regarding the role of ADAMs in sepsis, Horiuchi et al showed that ADAM17 inactivation in myeloid cells results in protection against endotoxin shock, ${ }^{62}$ although these effects were not observed in another infection model, i.e. acute lung inflammation. $^{63}$ Recently, it has also been shown that the rs653765 polymorphism in the ADAM10 promoter is associated with the development of severe sepsis in humans, indicating that ADAM10 might also be clinically important in sepsis. ${ }^{64}$

Especially ADAM17 is also implicated in rheumatoid arthritis, as both the expression of ADAM17 and TNF were up-regulated in arthritis-affected cartilage. ${ }^{65}$ Further supporting a role for ADAM1 7 in arthritis is the fact that selective inhibitors against ADAM17, including TMI-2 (Wyeth) and BMS-561392 (Bristol Myers Squibb), were effective in the treatment of mouse models of arthritis. ${ }^{66}$ Recently, Isozaki et al showed that ADAM10 is also over-expressed in the human rheumatoid arthritis synovial tissue, suggesting that besides ADAM17, ADAM10 might also be involved in the pathogenesis of rheumatoid arthritis. ${ }^{67}$

Alzheimer's disease is a neurodegenerative disease, which shares a common pathophysiological basis, i.e. hyperlipidaemia-induced endothelial injury and inflammation, with CVDs like atherosclerosis ${ }^{68}$ and is predominantly caused by $\beta$ - and $\gamma$-secretase-mediated production of toxic amyloid- $\beta$ peptides. The cleavage of the APP by $\alpha$-secretases (i.e. ADAMs) on the other hand has been shown to have neuroprotective functions in the development of Alzheimer's disease. Interestingly, $\beta$-amyloid was also found in atherosclerotic plaques ${ }^{69,70}$ and circulating levels of APP have been shown to correlate with CVD risk. ${ }^{71,72}$ Several ADAMs have been shown to possess $\alpha$-secretase activity and could therefore potentially contribute to neuro-protection ${ }^{73}$ and their role in brain inflammation has been nicely reviewed by Pruessmeyer and Ludwig. ${ }^{74}$ ADAM10 is the only ADAM family member that mediates a constitutive $\alpha$-secretase activity, ${ }^{75-77}$ whereas other ADAM proteases (e.g. ADAM8, 9 and 17) mediate a regulated secretase activity. Until now, it has been shown that ADAM10 can indeed be beneficial as this protease protected against amyloid- $\beta$ plaque formation by the cleavage of APP in a mouse model. ${ }^{78}$ This notion is further supported by a small clinical trial using the synthetic retinoid acitretin, which results in $\alpha$-secretase ADAM10 induction, showing enhanced APP processing in patients receiving acitretin. ${ }^{79}$ Furthermore, patients suffering from Alzheimer's disease express less ADAM10, again indicating that this protease could have protective functions. ${ }^{80}$ Interestingly, two polymorphisms in the ADAM12 gene have been significantly associated with late-onset Alzheimer's disease, although this protease does not possess $\alpha$-secretase activity suggesting a different mechanism of action. ${ }^{81}$

\section{Extracellular Vesicles and ADAMs}

In recent years, a highly interesting field of research emerged, investigating extracellular vesicles (e.g. microvesicles/micro-particles and exosomes) that are released from various cells under physiological and patho-physiological conditions. ${ }^{82}$ Micro-vesicles are derived from the cell membrane and are enriched with lipid rafts, while exosomes are smaller and of intracellular origin, enriched with tetraspanins. ${ }^{83}$ As mentioned before, lipid rafts and tetraspanins have also been shown to be key regulators of ADAMs activity. Canault et al indeed confirmed that ADAM17 is present in micro-vesicles released by human atherosclerotic plaques and actively contributes to the shedding of its substrates TNF and TNF receptor. ${ }^{84}$ Additionally, ADAM17 has been shown to be released in exosomes upon cell stimulation in monocytes and primary endothelial cells. ${ }^{85}$ Furthermore, it has been shown that ADAM10 is present in exosomes and can cleave adhesion molecules like L1 and CD44, ${ }^{86}$ while ADAM15 exists in exosomes released from human macrophages. ${ }^{87}$ As extracellular vesicles are considered to be a novel means of intercellular communication, modulating various target cell functions, it will be interesting to further evaluate the systemic effects that ADAMs released in extracellular vesicles can have in different pathologies. Moreover, as extracellular vesicle composition often reflects the phenotype of its parental cell, these vesicles could also serve as (circulating, i.e. non-invasive) biomarkers for CVD.

\section{ADAMs in Therapeutic and Diagnostic Approaches (Theranostics)}

As described, ADAMs (and their substrates) are associated with and causally related to CVDs, but also implicated in other pathologies like Alzheimer's disease and rheumatoid arthritis, rendering them interesting candidates for novel diagnostic or therapeutic tools ( - Fig. 1). The effects of ADAM activity, either being beneficial or detrimental, vary between different pathologies. Due to this discrepancy, in combination with the variety of ADAM proteases involved, the large list of associated substrates and the cell-specific effects, caution is warranted when developing ADAM-based therapies as unwanted side effects of ADAM inhibition seem almost inevitable. Therefore, inhibition should be very precise with respect to target-protease, -location and -timing. In recent years, research has focused on more specific approaches and developing appropriate inhibitors. As ADAM10 and ADAM17 look like the most suited candidates for targeting, we will focus on these two proteases.

The different levels of ADAM regulation provide several options for therapeutic targeting. The first option is to inhibit the expression of ADAMs, which, for example, has been showed to reduce inflammation by reducing ADAM10 and ADAM17 expression via peroxisome proliferator-activated 

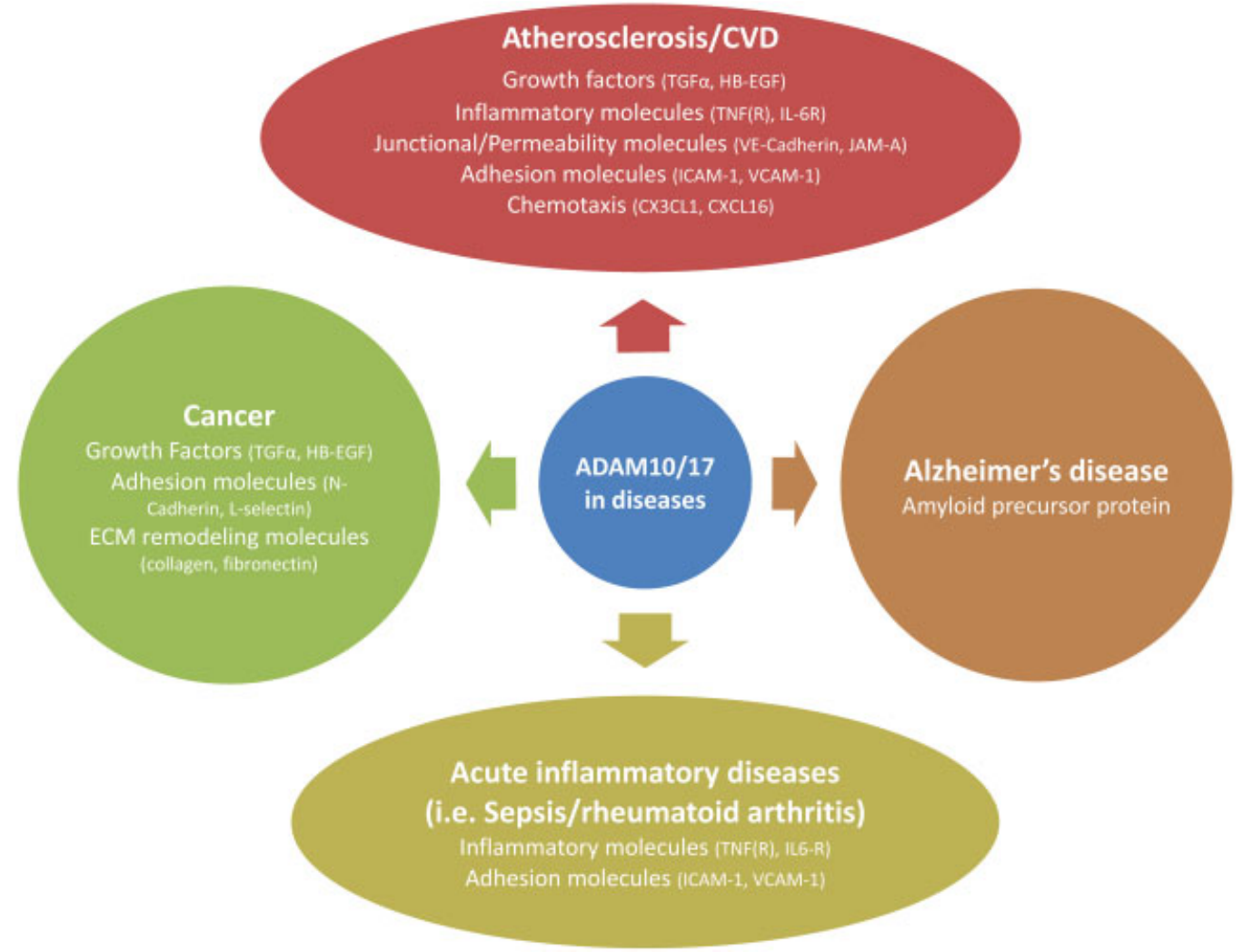

Fig. 1 Generalized overview of a disintegrin and metalloprotease (ADAM)10/17 influences in various pathologies. Depicted are selected main groups of molecules that are influenced by ADAM10/17 in different pathologies. Between brackets is for exemplary purposes, only a small selection of group members shown.

receptor activation. ${ }^{88}$ However, such targeting of course lacks any selectivity for substrates. Several hydroxamatebased inhibitors have already been developed to inhibit the active site of proteases, by inhibiting the zinc-ion binding. For example, the GI254023X compound inhibits ADAM10 100 -fold more potently than ADAM17, ${ }^{89}$ although still lacking specificity as it also weakly inhibits ADAM9 and several matrix metalloproteinases. ${ }^{90}$ Various other inhibitors have been developed, although until now no real ADAM-specific inhibition has been observed. ${ }^{91}$ Recently, Tape et al developed a specific ADAM17 inhibitor using a two-step phage display approach. ${ }^{92}$ The resulting cross-domain human antibody is a previously undescribed selective ADAM17 antagonist, providing a unique alternative to small-molecule metalloprotease inhibition. ${ }^{92}$ Besides the active zinc-binding domain, also a hyper-variable region called the exosite can come into contact with the substrate. Such exosites recognize specific glycosylation patterns on ADAM substrates and are therefore potential targets for substrate-specific targeting. Recently, inhibitors of these exosites of ADAM17 have been developed, showing substrate selectivity as TNF shedding can be blocked without affecting TGF or CX ${ }_{3}$ CL1 shedding. ${ }^{93}$ Recently, it has been shown that inactive rhomboid like protease (iRhom 2 ) is critically involved in the maturation of specifically ADAM17. 22,23 Interestingly, this iRhom2 involvement is, in the vascular system, mainly observed in leukocytes and can therefore be potentially used to more selectively target ADAM17 in inflammatory diseases. ${ }^{23,94}$ In contrast, ADAM10 maturation is not mediated by iRhom2, although recently also here some selective targeting opportunities emerged. Tspanc8 tetraspanins have been shown to differentially regulate the cleavage of ADAM10 substrates, as Tspan 15 was the only TspanC 8 member to be able to promote ADAM10-mediated N-cadherin cleavage, whereas Tspan14 distinctively reduced cleavage of the GPVI receptor. ${ }^{20,95}$ As many ADAMs are implicated in the physiological shedding of important proteins like growth factors, such substrate and cellular selectivity will be of great importance to develop suitable ADAM-based therapies. This is also highlighted by the discontinuation of several clinical trials targeting ADAM17 due to unspecific and unwanted side effects. ${ }^{66}$

Besides therapeutic targets, ADAMs could also serve as diagnostic as well as prognostic biomarkers ( - Table 2). Several reports do already suggest that several ADAMs may be used as biomarkers for cancer. ${ }^{96}$ High urinary ADAM12 levels, for example, were significantly correlated with the presence of breast cancer and bladder cancer. As a more prognostic marker, high levels of ADAM10/17 were even found to predict adverse outcome in patients with breast cancer. Until now, however, research into ADAMs as biomarkers has been limited, especially in the field of atherosclerosis and CVD. Several substrates of ADAM10/17 have already been proposed as biomarkers, although individually it does not appear to be strong enough to obtain a clear association/prediction. It is tempting to speculate that perhaps a panel of several ADAM substrates could increase the predictive and diagnostic potential. 
Table 2 ADAMs in diagnosis and therapy

\begin{tabular}{|l|l|l|}
\hline $\begin{array}{l}\text { ADAM } \\
\text { proteases }\end{array}$ & Main conclusion & Reference \\
\hline ADAM9 & Increased expression in prostate cancer is associated with shortened relapse-free survival & 98 \\
\hline ADAM12 & High urinary levels correlate with the presence of breast cancer and bladder cancer & 99,100 \\
\hline ADAM17 & High expression levels are predictive of a more adverse outcome in patients with breast cancer & 101 \\
\hline ADAM28 & High diagnostic accuracy of serum levels for non-small cell lung cancer & 102 \\
\hline
\end{tabular}

Abbreviation: ADAM, a disintegrin and metalloprotease.

\section{Conclusion}

It is clear that several ADAMs can play a crucial role in a large scale of pathologies, such as atherosclerosis. However, the regulation at post-translational level by differential trafficking and activation and the interaction of various ADAMs and their substrates is very complex and not yet fully elucidated. Especially, the precise in vivo and particularly cell-specific effects of ADAMs in the various pathologies still largely needs to be determined as only a few studies so far investigated this. Circulating levels of sADAM8 or a panel of ADAM substrates may become useful biomarkers for CVD, although specificity is an issue as their circulating levels will be affected in many inflammatory diseases and/or cancer. With the prospect of using ADAMs as therapeutic or diagnostic targets, the identification of more specific and perhaps even cell type-specific regulation modalities, such as the iRhom2, is critical. The presence of ADAMs in extracellular vesicles might also be useful in this regard, as this at least allows to detect circulating ADAMs derived from specific cell types. Therefore, future research should focus on elucidating such specificity to create new opportunities to develop suitable ADAM-based theranostics.

\section{Funding}

This work was supported by the Alexander von Humboldt Foundation to E.P.C.v.d.V., by the Deutsche Forschungsgemeinschaft (SFB 1123-A1), the German Centre for Cardiovascular Research (MHA VD 1.2), the European Research Council (ERC AdG ${ }^{\circ}$ 249929), the German Federal Ministry of Education and Research (grant number 01KU1213A), the Leducq Transatlantic Network CVGene(Fx) to C.W. and by the Netherlands Heart Foundation (Dr. E. Dekker grant 2012T079) to M.M.P.C.D.

Conflict of Interest

None.

\section{References}

1 Reiss K, Saftig P. The "a disintegrin and metalloprotease" (ADAM) family of sheddases: physiological and cellular functions. Semin Cell Dev Biol 2009;20(02):126-137

2 Edwards DR, Handsley MM, Pennington CJ. The ADAM metalloproteinases. Mol Aspects Med 2008;29(05):258-289
3 Bridges LC, Bowditch RD. ADAM-integrin interactions: potential integrin regulated ectodomain shedding activity. Curr Pharm Des 2005;11(07):837-847

4 Dreymueller D, Pruessmeyer J, Groth E, Ludwig A. The role of ADAM-mediated shedding in vascular biology. Eur J Cell Biol 2012;91(6-7):472-485

5 van der Vorst EP, Keijbeck AA, de Winther MP, Donners MM. A disintegrin and metalloproteases: molecular scissors in angiogenesis, inflammation and atherosclerosis. Atherosclerosis 2012;224(02):302-308

6 Zhang P, Shen M, Fernandez-Patron C, Kassiri Z. ADAMs family and relatives in cardiovascular physiology and pathology. J Mol Cell Cardiol 2016;93:186-199

7 Zunke F, Rose-John S. The shedding protease ADAM17: physiology and pathophysiology. Biochim Biophys Acta 2017;1864 (11 Pt B):2059-2070

8 Black RA, Rauch CT, Kozlosky CJ, et al. A metalloproteinase disintegrin that releases tumour-necrosis factor-alpha from cells. Nature 1997;385(6618):729-733

9 Dreymueller D, Ludwig A. Considerations on inhibition approaches for proinflammatory functions of ADAM proteases. Platelets 2017;28(04):354-361

10 Grötzinger J, Lorenzen I, Düsterhöft S. Molecular insights into the multilayered regulation of ADAM17: the role of the extracellular region. Biochim Biophys Acta 2017;1864(11 Pt B):2088-2095

11 Duffy MJ, McKiernan E, O'Donovan N, McGowan PM. The role of ADAMs in disease pathophysiology. Clin Chim Acta 2009;403 (1-2):31-36

12 Reiss K, Ludwig A, Saftig P. Breaking up the tie: disintegrin-like metalloproteinases as regulators of cell migration in inflammation and invasion. Pharmacol Ther 2006;111(03):985-1006

13 Sommer A, Kordowski F, Büch J, et al. Phosphatidylserine exposure is required for ADAM17 sheddase function. Nat Commun 2016;7:11523

14 Varshney P, Yadav V, Saini N. Lipid rafts in immune signalling: current progress and future perspective. Immunology 2016;149 (01):13-24

15 Tellier E, Canault M, Rebsomen L, et al. The shedding activity of ADAM17 is sequestered in lipid rafts. Exp Cell Res 2006;312(20): 3969-3980

16 Ebsen H, Lettau M, Kabelitz D, Janssen O. Subcellular localization and activation of ADAM proteases in the context of FasL shedding in T lymphocytes. Mol Immunol 2015;65(02):416-428

17 Xu D, Sharma C, Hemler ME. Tetraspanin12 regulates ADAM10dependent cleavage of amyloid precursor protein. FASEB J 2009; 23(11):3674-3681

18 Arduise C, Abache T, Li L, et al. Tetraspanins regulate ADAM10mediated cleavage of TNF-alpha and epidermal growth factor. J Immunol 2008;181(10):7002-7013

19 Haining EJ, Yang J, Bailey RL, et al. The TspanC8 subgroup of tetraspanins interacts with A disintegrin and metalloprotease 10 (ADAM10) and regulates its maturation and cell surface expression. J Biol Chem 2012;287(47):39753-39765 
20 Noy PJ, Yang J, Reyat JS, et al. TspanC8 tetraspanins and a disintegrin and metalloprotease 10 (ADAM10) interact via their extracellular regions: evidence for distinct binding mechanisms for different TspanC8 proteins. J Biol Chem 2016;291(07):3145-3157

21 Maney SK, Mcllwain DR, Polz R, et al. Deletions in the cytoplasmic domain of iRhom 1 and iRhom 2 promote shedding of the TNF receptor by the protease ADAM17. Sci Signal 2015;8(401):ra109

22 Adrain C, Zettl M, Christova Y, Taylor N, Freeman M. Tumor necrosis factor signaling requires iRhom 2 to promote trafficking and activation of TACE. Science 2012;335(6065):225-228

23 Mcllwain DR, Lang PA, Maretzky T, et al. iRhom2 regulation of TACE controls TNF-mediated protection against Listeria and responses to LPS. Science 2012;335(6065):229-232

24 Donners MM, Wolfs IM, Olieslagers S, et al. A disintegrin and metalloprotease 10 is a novel mediator of vascular endothelial growth factor-induced endothelial cell function in angiogenesis and is associated with atherosclerosis. Arterioscler Thromb Vasc Biol 2010;30(11):2188-2195

25 Koenen RR, Pruessmeyer J, Soehnlein O, et al. Regulated release and functional modulation of junctional adhesion molecule $A$ by disintegrin metalloproteinases. Blood 2009;113(19):4799-4809

26 Schulz B, Pruessmeyer J, Maretzky T, et al. ADAM10 regulates endothelial permeability and T-Cell transmigration by proteolysis of vascular endothelial cadherin. Circ Res 2008;102(10):1192-1201

27 Matthews AL, Noy PJ, Reyat JS, Tomlinson MG. Regulation of A disintegrin and metalloproteinase (ADAM) family sheddases ADAM10 and ADAM17: the emerging role of tetraspanins and rhomboids. Platelets 2017;28(04):333-341

28 Hundhausen C, Schulte A, Schulz B, et al. Regulated shedding of transmembrane chemokines by the disintegrin and metalloproteinase 10 facilitates detachment of adherent leukocytes. J Immunol 2007;178(12):8064-8072

29 Ludwig A, Weber C. Transmembrane chemokines: versatile 'special agents' in vascular inflammation. Thromb Haemost 2007;97(05):694-703

30 Garton KJ, Gough PJ, Philalay J, et al. Stimulated shedding of vascular cell adhesion molecule 1 (VCAM- 1 ) is mediated by tumor necrosis factor-alpha-converting enzyme (ADAM 17). J Biol Chem 2003;278(39):37459-37464

31 Tsakadze NL, Sithu SD, Sen U, English WR, Murphy G, D'Souza SE. Tumor necrosis factor-alpha-converting enzyme (TACE/ADAM17) mediates the ectodomain cleavage of intercellular adhesion molecule-1 (ICAM-1). J Biol Chem 2006;281(06):3157-3164

32 Pasqualon T, Pruessmeyer J, Weidenfeld S, et al. A transmembrane C-terminal fragment of syndecan- 1 is generated by the metalloproteinase ADAM17 and promotes lung epithelial tumor cell migration and lung metastasis formation. Cell Mol Life Sci 2015;72(19):3783-3801

33 Pruessmeyer J, Martin C, Hess FM, et al. A disintegrin and metalloproteinase 17 (ADAM17) mediates inflammationinduced shedding of syndecan- 1 and -4 by lung epithelial cells. J Biol Chem 2010;285(01):555-564

34 Demerath E, Towne B, Blangero J, Siervogel RM. The relationship of soluble ICAM-1, VCAM-1, P-selectin and E-selectin to cardiovascular disease risk factors in healthy men and women. Ann Hum Biol 2001;28(06):664-678

35 Lazo M, Halushka MK, Shen L, et al. Soluble receptor for advanced glycation end products and the risk for incident heart failure: the Atherosclerosis Risk in Communities Study. Am Heart J 2015;170 (05):961-967

36 Lobbes MB, Lutgens E, Heeneman S, et al. Is there more than Creactive protein and fibrinogen? The prognostic value of soluble CD40 ligand, interleukin-6 and oxidized low-density lipoprotein with respect to coronary and cerebral vascular disease. Atherosclerosis 2006;187(01):18-25

37 Rizza S, Copetti M, Cardellini M, et al. A score including ADAM17 substrates correlates to recurring cardiovascular event in subjects with atherosclerosis. Atherosclerosis 2015;239(02):459-464
38 Li Y, Liao F, Yin XJ, et al. An association study on ADAM10 promoter polymorphisms and atherosclerotic cerebral infarction in a Chinese population. CNS Neurosci Ther 2013;19(10): 785-794

39 van der Vorst EP, Jeurissen M, Wolfs IM, et al. Myeloid A disintegrin and metalloproteinase domain 10 deficiency modulates atherosclerotic plaque composition by shifting the balance from inflammation toward fibrosis. Am J Pathol 2015;185 (04):1145-1155

40 Holdt LM, Thiery J, Breslow JL, Teupser D. Increased ADAM17 mRNA expression and activity is associated with atherosclerosis resistance in LDL-receptor deficient mice. Arterioscler Thromb Vasc Biol 2008;28(06):1097-1103

41 Zheng DY, Zhao J, Yang JM, Wang M, Zhang XT. Enhanced ADAM17 expression is associated with cardiac remodeling in rats with acute myocardial infarction. Life Sci 2016;151:61-69

42 Fan D, Takawale A, Shen M, et al. Cardiomyocyte a disintegrin and metalloproteinase 17 (ADAM17) is essential in post-myocardial infarction repair by regulating angiogenesis. Circ Heart Fail 2015;8(05):970-979

43 Chute M, Jana S, Kassiri Z. Disintegrin and metalloproteinases (ADAMs and ADAM-TSs), the emerging family of proteases in heart physiology and pathology. Curr Opin Physiol 2018; $1: 34-45$

44 Canault M, Peiretti F, Kopp F, et al. The TNF alpha converting enzyme (TACE/ADAM17) is expressed in the atherosclerotic lesions of apolipoprotein E-deficient mice: possible contribution to elevated plasma levels of soluble TNF alpha receptors. Atherosclerosis 2006;187(01):82-91

45 Zhao X, Kong J, Zhao Y, et al. Gene silencing of TACE enhances plaque stability and improves vascular remodeling in a rabbit model of atherosclerosis. Sci Rep 2015;5:17939

46 Chalaris A, Adam N, Sina C, et al. Critical role of the disintegrin metalloprotease ADAM17 for intestinal inflammation and regeneration in mice. J Exp Med 2010;207(08):1617-1624

47 Nicolaou A, Zhao Z, Northoff BH, et al. Adam17 deficiency promotes atherosclerosis by enhanced TNFR2 signaling in mice. Arterioscler Thromb Vasc Biol 2017;37(02):247-257

48 Nicolaou A, Northoff BH, Zhao Z, et al. The ADAM17 metalloproteinase maintains arterial elasticity. Thromb Haemost 2018; 118(01):210-213

49 van der Vorst EP, Zhao Z, Rami M, et al. Contrasting effects of myeloid and endothelial ADAM17 on atherosclerosis development. Thromb Haemost 2017;117(03):644-646

50 Oksala N, Levula M, Airla N, et al. ADAM-9, ADAM-15, and ADAM17 are upregulated in macrophages in advanced human atherosclerotic plaques in aorta and carotid and femoral arteriesTampere vascular study. Ann Med 2009;41(04):279-290

51 Bültmann A, Li Z, Wagner S, et al. Loss of protease activity of ADAM15 abolishes protective effects on plaque progression in atherosclerosis. Int J Cardiol 2011;152(03):382-385

52 Sun C, Wu MH, Guo M, Day ML, Lee ES, Yuan SY. ADAM15 regulates endothelial permeability and neutrophil migration via Src/ERK1/2 signalling. Cardiovasc Res 2010;87(02):348-355

53 Sun C, Wu MH, Lee ES, Yuan SY. A disintegrin and metalloproteinase 15 contributes to atherosclerosis by mediating endothelial barrier dysfunction via Src family kinase activity. Arterioscler Thromb Vasc Biol 2012;32(10):2444-2451

54 Holloway JW, Laxton RC, Rose-Zerilli MJ, et al. ADAM33 expression in atherosclerotic lesions and relationship of ADAM33 gene variation with atherosclerosis. Atherosclerosis 2010;211(01): 224-230

55 Levula M, Airla N, Oksala N, et al. ADAM8 and its single nucleotide polymorphism $2662 \mathrm{~T} / \mathrm{G}$ are associated with advanced atherosclerosis and fatal myocardial infarction: Tampere Vascular study. Ann Med 2009;41(07):497-507

56 Raitoharju E, Seppälä I, Levula M, et al. Common variation in the ADAM8 gene affects serum SADAM8 concentrations and the risk 
of myocardial infarction in two independent cohorts. Atherosclerosis 2011;218(01):127-133

57 Theodorou K, van der Vorst EPC, Gijbels MJ, et al. Whole body and hematopoietic ADAM8 deficiency does not influence advanced atherosclerotic lesion development, despite its association with human plaque progression. Sci Rep 2017;7(01):11670

58 Menghini R, Fiorentino L, Casagrande V, Lauro R, Federici M. The role of ADAM17 in metabolic inflammation. Atherosclerosis 2013;228(01):12-17

59 Kraakman MJ, Kammoun HL, Allen TL, et al. Blocking IL-6 transsignaling prevents high-fat diet-induced adipose tissue macrophage recruitment but does not improve insulin resistance. Cell Metab 2015;21(03):403-416

60 Schuett H, Oestreich R, Waetzig GH, et al. Transsignaling of interleukin-6 crucially contributes to atherosclerosis in mice. Arterioscler Thromb Vasc Biol 2012;32(02):281-290

61 Lee AC, Lam JK, Shiu SW, Wong Y, Betteridge DJ, Tan KC. Serum level of soluble receptor for advanced glycation end products is associated with a disintegrin and metalloproteinase 10 in type 1 diabetes. PLoS One 2015;10(09):e0137330

62 Horiuchi K, Kimura T, Miyamoto T, et al. Cutting edge: TNFalpha-converting enzyme (TACE/ADAM17) inactivation in mouse myeloid cells prevents lethality from endotoxin shock. J Immunol 2007;179(05):2686-2689

63 Pruessmeyer J, Hess FM, Alert $\mathrm{H}$, et al. Leukocytes require ADAM10 but not ADAM17 for their migration and inflammatory recruitment into the alveolar space. Blood 2014;123(26): 4077-4088

64 Cui L, Gao Y, Xie Y, et al. An ADAM10 promoter polymorphism is a functional variant in severe sepsis patients and confers susceptibility to the development of sepsis. Crit Care 2015;19:73

65 Patel IR, Attur MG, Patel RN, et al. TNF-alpha convertase enzyme from human arthritis-affected cartilage: isolation of cDNA by differential display, expression of the active enzyme, and regulation of TNF-alpha. J Immunol 1998;160(09):4570-4579

66 Moss ML, Sklair-Tavron L, Nudelman R. Drug insight: tumor necrosis factor-converting enzyme as a pharmaceutical target for rheumatoid arthritis. Nat Clin Pract Rheumatol 2008;4(06): 300-309

67 Isozaki T, Rabquer BJ, Ruth JH, Haines GK III, Koch AE. ADAM-10 is overexpressed in rheumatoid arthritis synovial tissue and mediates angiogenesis. Arthritis Rheum 2013;65(01):98-108

68 Akinyemi RO, Mukaetova-Ladinska EB, Attems J, Ihara M, Kalaria RN. Vascular risk factors and neurodegeneration in ageing related dementias: Alzheimer's disease and vascular dementia. Curr Alzheimer Res 2013;10(06):642-653

69 Bucerius J, Barthel H, Tiepolt S, et al. Feasibility of in vivo ${ }^{18} \mathrm{~F}-$ florbetaben PET/MR imaging of human carotid amyloid- $\beta$. Eur J Nucl Med Mol Imaging 2017;44(07):1119-1128

70 Jans DM, Martinet W, Van De Parre TJ, et al. Processing of amyloid precursor protein as a biochemical link between atherosclerosis and Alzheimer's disease. Cardiovasc Hematol Disord Drug Targets 2006;6(01):21-34

71 Lee PH, Bang OY, Hwang EM, et al. Circulating beta amyloid protein is elevated in patients with acute ischemic stroke. J Neural Transm (Vienna) 2005;112(10):1371-1379

72 Stamatelopoulos K, Sibbing D, Rallidis LS, et al. Amyloid-beta (1-40) and the risk of death from cardiovascular causes in patients with coronary heart disease. J Am Coll Cardiol 2015;65(09):904-916

73 Asai M, Hattori C, Szabó B, et al. Putative function of ADAM9, ADAM10, and ADAM17 as APP alpha-secretase. Biochem Biophys Res Commun 2003;301(01):231-235

74 Pruessmeyer J, Ludwig A. The good, the bad and the ugly substrates for ADAM10 and ADAM17 in brain pathology, inflammation and cancer. Semin Cell Dev Biol 2009;20(02):164-174

75 Jorissen E, Prox J, Bernreuther C, et al. The disintegrin/metalloproteinase ADAM10 is essential for the establishment of the brain cortex. J Neurosci 2010;30(14):4833-4844
76 Kuhn PH, Wang H, Dislich B, et al. ADAM10 is the physiologically relevant, constitutive alpha-secretase of the amyloid precursor protein in primary neurons. EMBO J 2010;29(17):3020-3032

77 Lammich S, Kojro E, Postina R, et al. Constitutive and regulated alpha-secretase cleavage of Alzheimer's amyloid precursor protein by a disintegrin metalloprotease. Proc Natl Acad Sci U S A 1999;96(07):3922-3927

78 Postina R, Schroeder A, Dewachter I, et al. A disintegrin-metalloproteinase prevents amyloid plaque formation and hippocampal defects in an Alzheimer disease mouse model. J Clin Invest 2004;113(10):1456-1464

79 Endres K, Fahrenholz F, Lotz J, et al. Increased CSF APPs- $\alpha$ levels in patients with Alzheimer disease treated with acitretin. Neurology 2014;83(21):1930-1935

80 Colciaghi F, Borroni B, Pastorino L, et al. [alpha]-Secretase ADAM10 as well as [alpha]APPs is reduced in platelets and CSF of Alzheimer disease patients. Mol Med 2002;8(02):67-74

81 Harold D, Jehu L, Turic D, et al. Interaction between the ADAM12 and SH3MD1 genes may confer susceptibility to late-onset Alzheimer's disease. Am J Med Genet B Neuropsychiatr Genet 2007;144B(04):448-452

82 Mause SF, Weber C. Microparticles: protagonists of a novel communication network for intercellular information exchange. Circ Res 2010;107(09):1047-1057

83 Théry C, Ostrowski M, Segura E. Membrane vesicles as conveyors of immune responses. Nat Rev Immunol 2009;9(08):581-593

84 Canault M, Leroyer AS, Peiretti F, et al. Microparticles of human atherosclerotic plaques enhance the shedding of the tumor necrosis factor-alpha converting enzyme/ADAM17 substrates, tumor necrosis factor and tumor necrosis factor receptor-1. Am J Pathol 2007;171(05):1713-1723

85 Groth E, Pruessmeyer J, Babendreyer A, et al. Stimulated release and functional activity of surface expressed metalloproteinase ADAM17 in exosomes. Biochim Biophys Acta 2016;1863(11): 2795-2808

86 Stoeck A, Keller S, Riedle S, et al. A role for exosomes in the constitutive and stimulus-induced ectodomain cleavage of L1 and CD44. Biochem J 2006;393(Pt 3):609-618

87 Lee HD, Koo BH, Kim YH, Jeon OH, Kim DS. Exosome release of ADAM15 and the functional implications of human macrophagederived ADAM15 exosomes. FASEB J 2012;26(07):3084-3095

88 Tripathy D, Daniele G, Fiorentino TV, et al. Pioglitazone improves glucose metabolism and modulates skeletal muscle TIMP-3TACE dyad in type 2 diabetes mellitus: a randomised, doubleblind, placebo-controlled, mechanistic study. Diabetologia 2013; 56(10):2153-2163

89 Hoettecke N, Ludwig A, Foro S, Schmidt B. Improved synthesis of ADAM10 inhibitor GI254023X. Neurodegener Dis 2010;7(04): 232-238

90 Moss ML, Rasmussen FH, Nudelman R, Dempsey PJ, Williams J. Fluorescent substrates useful as high-throughput screening tools for ADAM9. Comb Chem High Throughput Screen 2010; 13(04):358-365

91 Ludwig A, Hundhausen C, Lambert MH, et al. Metalloproteinase inhibitors for the disintegrin-like metalloproteinases ADAM10 and ADAM17 that differentially block constitutive and phorbol ester-inducible shedding of cell surface molecules. Comb Chem High Throughput Screen 2005;8(02):161-171

92 Tape CJ, Willems SH, Dombernowsky SL, et al. Cross-domain inhibition of TACE ectodomain. Proc Natl Acad Sci U S A 2011;108 (14):5578-5583

93 Knapinska AM, Dreymuller D, Ludwig A, et al. SAR studies of exosite-binding substrate-selective inhibitors of a disintegrin and metalloprotease 17 (ADAM17) and application as selective in vitro probes. J Med Chem 2015;58(15):5808-5824

94 Maretzky T, Mcllwain DR, Issuree PD, et al. iRhom2 controls the substrate selectivity of stimulated ADAM17-dependent ectodomain shedding. Proc Natl Acad Sci U S A 2013;110(28):11433-11438 
95 Jouannet S, Saint-Pol J, Fernandez L, et al. TspanC8 tetraspanins differentially regulate the cleavage of ADAM10 substrates, Notch activation and ADAM10 membrane compartmentalization. Cell Mol Life Sci 2016;73(09):1895-1915

96 Duffy MJ, Mullooly M, O'Donovan N, et al. The ADAMs family of proteases: new biomarkers and therapeutic targets for cancer? Clin Proteomics 2011;8(01):9

97 Figarska SM, Vonk JM, van Diemen CC, Postma DS, Boezen HM. ADAM33 gene polymorphisms and mortality. A prospective cohort study. PLoS One 2013;8(07):e67768

98 Fritzsche FR, Jung M, Tölle A, et al. ADAM9 expression is a significant and independent prognostic marker of PSA relapse in prostate cancer. Eur Urol 2008;54(05):1097-1106
99 Fröhlich C, Albrechtsen R, Dyrskjøt L, Rudkjaer L, Ørntoft TF, Wewer UM. Molecular profiling of ADAM12 in human bladder cancer. Clin Cancer Res 2006;12(24):7359-7368

100 Roy R, Wewer UM, Zurakowski D, Pories SE, Moses MA. ADAM 12 cleaves extracellular matrix proteins and correlates with cancer status and stage. J Biol Chem 2004;279(49): 51323-51330

101 McGowan PM, McKiernan E, Bolster F, et al. ADAM-17 predicts adverse outcome in patients with breast cancer. Ann Oncol 2008; 19(06):1075-1081

102 Kuroda H, Mochizuki S, Shimoda M, et al. ADAM28 is a serological and histochemical marker for non-small-cell lung cancers. Int J Cancer 2010;127(08):1844-1856 